\title{
Editorial to the Special Issue "Selected Papers from the 17th Russian Gravitational Conference-International Conference on Gravitation, Cosmology and Astrophysics (RUSGRAV-17)"
}

\author{
Vladimir M. Mostepanenko ${ }^{1,2,3 * \mathbb{D}}$, Alexei A. Starobinsky ${ }^{4,5}$ and Elena N. Velichko ${ }^{2}$ \\ 1 Central Astronomical Observatory at Pulkovo of the Russian Academy of Sciences, \\ 196140 Saint Petersburg, Russia \\ 2 Institute of Physics, Nanotechnology and Telecommunications, \\ Peter the Great Saint Petersburg Polytechnic University, 195251 Saint Petersburg, Russia; \\ velichko_en@spbstu.ru \\ Kazan Federal University, 420008 Kazan, Russia \\ 4 Landau Institute for Theoretical Physics, 119334 Moscow, Russia; alstar@landau.ac.ru \\ 5 Bogoliubov Laboratory of Theoretical Physics, Joint Institute for Nuclear Research, 141980 Dubna, Russia \\ * Correspondence: vmostepa@gmail.com
}

check for updates

Citation: Mostepanenko, V.M.; Starobinsky, A.A.; Velichko, E.N. Editorial to the Special Issue

"Selected Papers from the 17th

Russian Gravitational

Conference-International

Conference on Gravitation,

Cosmology and Astrophysics

(RUSGRAV-17)". Universe 2021, 7, 296.

https: / / doi.org/10.3390/

universe7080296

Received: 6 August 2021

Accepted: 8 August 2021

Published: 12 August 2021

Publisher's Note: MDPI stays neutral with regard to jurisdictional claims in published maps and institutional affiliations.

Copyright: (c) 2021 by the authors. Licensee MDPI, Basel, Switzerland. This article is an open access article distributed under the terms and conditions of the Creative Commons Attribution (CC BY) license (https:// creativecommons.org/licenses/by/ $4.0 /)$.
This Special Issue consists of selected papers reflecting the plenary and sectional talks presented at the 17th Russian Gravitational Conference-International Conference on Gravitation, Cosmology and Astrophysics (RUSGRAV-17). The aim of this Conference and Special Issue is to present important new results regarding classical and quantum gravity, cosmology, relativistic astrophysics, physics of black holes, neutron stars, dark matter and dark energy, and possible unification schemes of particle and gravitational theories. Although the listed topics are rather diverse, all of them are concentrated around the greatest problem of modern physics, namely, what is the origin, structure, and evolution of our Universe. During the last decades, great progress has been made in the resolution of this fundamental problem, but many important questions remain unanswered. We still do not have a clear understanding of the very early stages of the Universe evolution, nor do we know what is the nature of dark matter and dark energy, which contribute the major part of the Universe energy. Moreover, the mechanisms of huge outputs of energy from remote astrophysical objects remain unknown. These global problems, whose resolution might demand some changes in our fundamental concepts, are supplemented by a great number of questions regarding the models of inflation, physical effects in black holes and different predictions of gravitational theories alternative to Einstein's general relativity theory.

In accordance to the main subjects discussed at RUSGRAV-17, selected papers included in the Special Issue can be conditionally attributed to classical gravity, physics of black holes and wormholes, cosmology, alternative gravity theories, quantum gravity, and to relativistic astrophysics.

Several articles of this Special Issue are devoted to different problems of classical gravity. An impact of extra symmetries on the exact solutions of general relativity is discussed in [1]. Article [2] considers the problem of universal constants and natural systems of units in the spacetime with an arbitrary number of dimensions. The concept of energy-momentum pseudotensor in generally covariant theories of gravity and the possibility of negative energy in generalized Vaidya spacetime are analyzed in [3,4]. We emphasize that the talk of Dr. Vertogradov reflected in the article [4] was awarded by the Organizing Committee of the Conference as the best talk presented by young scientist. Articles [5-7] are devoted to a comparison of gravitational and light frequency shifts, gravitational interaction in a null string gas, and to spinors in the cylindrically symmetric spacetime, respectively.

During the last years, much attention was devoted to investigation of black holes and wormholes. It should be noted that black holes, which were first predicted theoretically in 
the framework of Einstein's general relativity theory as some hypothetical objects, are now observed in the Universe and actively investigated by astrophysicists. It is well known that the spherically symmetric solutions of Einstein equations describing black holes are singular. The possibilities to find regular static solutions to Einstein equations for a spherically symmetric distribution of matter with no restriction on a total mass is discussed in $[8,9]$. In articles $[10,11]$ the collapsing wormholes sustained by dustlike matter and a discrete version of the Schwarzschild solution are considered, respectively. Null and timelike geodesics near the throats of phantom scalar field wormholes are investigated in [12]. Some other problems in the physics of black holes, such as matter accretion versus semiclassical bounce in the Schwarzschild interior [13], a description of black holes in terms of the axiomatic set theory [14], and an analysis of black hole formation in $(2+1)$-dimensional spacetime with the negative cosmological constant due to the collapse of a dust shell [15] are also discussed.

The largest scale application of gravitational theory is in cosmology which investigates the origin, structure, and evolution of the Universe. Modern cosmology is based on the solutions to Einstein equations found by Alexander Friedmann in 1922 which describe the expanding Universe. Some issues on the foundations of modern cosmology, gravitation and quantum physics are considered in review [16]. As was already mentioned above, the major part of the Universe energy avoids immediate observation through strong, electromagnetic and weak interactions and is seen through gravity only. According to one of the proposed approaches, dark matter may consist of light pseudoscalar particles called axions. In this connection, article [17] discusses a question: Is the axionic dark matter an equilibrium system? Several articles investigate gravitational radiation as the bremsstrahlung of superheavy particles in the early Universe [18], energy conservation law in the closed Universe in connection with a concept of the proper time [19], and the possibility of applying the Copenhagen interpretation to quantum cosmology [20]. The cosmological constant problem in the super-early Universe and the collision of relativistic shock waves and its impact on the large scale structure of the Universe are considered in [21,22], respectively. Articles [23,24] are devoted to exact and slow-roll solutions for exponential power-law inflation and to a qualitative analysis of the dynamics of twocomponent chiral cosmological model. Article [25] discusses a relationship between the $5 \mathrm{D}$ theory and cosmology.

The alternative gravity theories have a long history. The first of them have been developed very soon after the creation of Einstein's general relativity theory. Nowadays the work in the field of alternative gravity theories is motivated by existing problems which remain unresolved in the framework of general relativity theory for a long time. Among them one could mention the problems of dark matter and dark energy, as well as a failure of all attempts to create consistent quantum theory of gravity based on general relativity theory. Scale-dependent gravity in the superfluid vacuum theory is considered in [26] as a possible alternative to dark matter and dark energy. Three articles are devoted to quark stars in massive Brans-Dicke gravity with Tolman-Kuchowicz spacetime [27], non-relativistic limit of embedding gravity as general relativity with dark matter [28], and to the formation and clustering of primordial black holes in Brans-Dicke theory [29]. The hybrid metric-Palatini gravity in the case of regular stringlike configurations and gravity with higher derivatives in D-dimensions are discussed in [30,31], respectively. Finally, the gravitational interaction in the unified scalar field theory is considered in [32].

As mentioned above, we are still lacking fully consistent theory of quantized gravitational field. However, during the last decades an important progress has been made in quantum field theory in curved spacetime. It is some kind of tradition that both these scientific directions are often referred to as quantum gravity. In this Special Issue, the vacuum quantum effects in braneworlds on anti-de Sitter bulk are considered in [33]. Article [34] is devoted to a consideration of inherent relation between the Higgs mechanism and spacetime symmetry. The state of the art in constraining axion-to-nucleon coupling and non-Newtonian gravity from laboratory experiments in reviewed in [35]. 
The radiation from an inertial mirror horizon and the gravitational interaction of cosmic string with spinless particle are analyzed in [36,37], respectively. The other problems of quantum gravity, such as quantization of gravitationally bound systems [38], a comparison between Einstein's geometrical and Feynman's quantum-field-theoretical approaches to gravitational physics [39], and a crucial role of gravity in the formation of elementary particles [40], are also investigated.

With creation of powerful instruments and space programs, astrophysics has now become an important branch of both fundamental and applied science based on the solid foundation of gravitational and particle physics. Modern astrophysics investigates not only stars and planets, but also an interstellar medium, including dark matter and dark energy, the microwave background radiation, gravitational waves, galaxies, and the large-scale structure of the Universe. Some of these subjects were touched upon at the RUSGRAV-17 Conference and are reflected in this Special Issue. Thus, the density operator approach to turbulent flows in plasma and atmospheric fluids [41] and the line-of-sight analysis of spatial distribution of galaxies in the COSMOS2015 catalogue [42] are considered. Articles $[43,44]$ discuss a question of whether the object OJ 287 is a single supermassive black hole and the resonant effect for a Breit-Wheeler process in the field of a X-ray pulsar, respectively. Some other articles included in this Special Issue are devoted to relativistic effects in the orbital motion of S-stars at the galactic center [45], the resonant production of an ultrarelativistic electron-positron pair at the gamma quantum scattering [46], visible shapes of the black holes M87* and SgrA* [47], and the resonant effects in a photoproduction of ultrarelativistic electron-positron pairs on a nucleus [48]. Possible wormhole candidates in active galactic nuclei [49] and resonant effect of an annihilation channel in the interaction between an ultrarelativistic electron and a positron [50] are also discussed. Finally, article [51] considers the intensity and polarization of radiation emerging from a rotating accretion disc with increasing height and article [52] - the resonant ultrarelativistic electron-positron pair production by high-energy electrons in the field of a X-ray pulsar.

The above articles reflect the present state of the art in the already resolved and still unresolved problems in the field of gravitation, cosmology and astrophysics. The Guest Editors of this Special Issue hope that it will be useful for further progress and stimulate new important research directions.

Funding: The work of V.M.M. was partially supported by the Peter the Great Saint Petersburg Polytechnic University in the framework of the Russian state assignment for basic research (project N FSEG-2020-0024). V.M.M. was also partially funded by the Russian Foundation for Basic Research grant number 19-02-00453 A. A.A.S. was partly supported by the project number 0033-2019-0005 of the Russian Ministry of Science and Higher Education. E.N.V. was supported by the Russian Science Foundation under Grant No. 21-72-20029.

Institutional Review Board Statement: Not applicable.

Informed Consent Statement: Not applicable.

Data Availability Statement: Not applicable.

Acknowledgments: V.M.M. is grateful for partial support by the Russian Government Program of Competitive Growth of Kazan Federal University.

Conflicts of Interest: The authors declare no conflict of interest.

\section{References}

1. Beesham, A.; Makhanya, F. How Extra Symmetries Affect Solutions in General Relativity. Universe 2020, 6, 170. [CrossRef]

2. Sheykin, A.; Manida, S. Universal Constants and Natural Systems of Units in a Spacetime of Arbitrary Dimension. Universe 2020, 6, 166. [CrossRef]

3. Ilin, R.; Paston, S. Energy-Momentum Pseudotensor and Superpotential for Generally Covariant Theories of Gravity of General Form. Universe 2020, 6, 173. [CrossRef]

4. Vertogradov, V. The Negative Energy in Generalized Vaidya Spacetime. Universe 2020, 6, 155. [CrossRef]

5. Baranov, A.; Ermak, S.; Lozov, R.; Semenov, V. Comparison of Gravitational and Light Frequency Shifts in Rubidium Atomic Clock. Universe 2021, 7, 3. [CrossRef] 
6. Lelyakov, A. Gravitational Interaction in a Null String Gas and Its Possible Consequences. Universe 2020, 6, 142. [CrossRef]

7. Saha, B. Spinors in Cylindrically Symmetric Space-Time. Universe 2020, 6, 152. [CrossRef]

8. Meierovich, B.E. Guessing the Riddle of a Black Hole. Universe 2020, 6, 113. [CrossRef]

9. Radinschi, I.; Grammenos, T.; Rahaman, F.; Cazacu, M.-M.; Spanou, A.; Chakraborty J. On the Energy of a Non-Singular Black Hole Solution Satisfying the Weak Energy Condition. Universe 2020, 6, 169. [CrossRef]

10. Kashargin, P.E.; Sushkov, S.V. Collapsing Wormholes Sustained by Dustlike Matter. Universe 2020, 6, 186. [CrossRef]

11. Khatsymovsky, V. On the Discrete Version of the Schwarzschild Problem. Universe 2020, 6, 185. [CrossRef]

12. Potashov, I.; Tchemarina, J.; Tsirulev, A. Null and Timelike Geodesics near the Throats of Phantom Scalar Field Wormholes. Universe 2020, 6, 183. [CrossRef]

13. Bronnikov, K.; Bolokhov, S.; Skvortsova, M. Matter Accretion Versus Semiclassical Bounce in Schwarzschild Interior. Universe 2020, 6, 178. [CrossRef]

14. Król, J.; Klimasara, P. Black Holes and Complexity via Constructible Universe. Universe 2020, 6, 198. [CrossRef]

15. Andrianov, A.A.; Starodubtsev, A.; Elmahalawy, Y. Quantum Analysis of BTZ Black Hole Formation Due to the Collapse of a Dust Shell. Universe 2020, 6, 201. [CrossRef]

16. Elizalde, E. Some Issues on the Foundations of Modern Cosmology, Gravitation and Quantum Physics. Universe 2020, 6, 189. [CrossRef]

17. Balakin, A.B.; Shakirzyanov, A.F. Is the Axionic Dark Matter an Equilibrium System? Universe 2020, 6, 192. [CrossRef]

18. Grib, A.A.; Pavlov, Y.V. Gravitational Radiation as the Bremsstrahlung of Superheavy Particles in the Early Universe. Universe 2020, 6, 188. [CrossRef]

19. Gorobey, N.; Lukyanenko, A.; Drozdov, P. Energy Conservation Law in the Closed Universe and a Concept of the Proper Time. Universe 2020, 6, 174. [CrossRef]

20. Shestakova, T.P. Is the Copenhagen Interpretation Inapplicable to Quantum Cosmology? Universe 2020, 6, 128. [CrossRef]

21. Babourova, O.; Frolov, B. The Solution of the Cosmological Constant Problem: The Cosmological Constant Exponential Decrease in the Super-Early Universe. Universe 2020, 6, 230. [CrossRef]

22. Golubiatnikov, A.; Lyuboshits, D. On the Collision of Relativistic Shock Waves and the Large Scale Structure of the Universe. Universe 2020, 6, 214. [CrossRef]

23. Fomin, I.; Chervon, S. Exact and Slow-Roll Solutions for Exponential Power-Law Inflation Connected with Modified Gravity and Observational Constraints. Universe 2020, 6, 199. [CrossRef]

24. Zhuravlev, V.; Chervon, S. Qualitative Analysis of the Dynamics of a Two-Component Chiral Cosmological. Universe 2020, 6, 195. [CrossRef]

25. Aliyev, B.G. Generalized Rest Mass and Dirac's Monopole in 5D Theory and Cosmology. Universe 2021, 7, 295. [CrossRef]

26. Zloshchastiev, K.G. An Alternative to Dark Matter and Dark Energy: Scale-Dependent Gravity in Superfluid Vacuum Theory. Universe 2020, 6, 180. [CrossRef]

27. Majid, A.; Sharif, M. Quark Stars in Massive Brans-Dicke Gravity with Tolman-Kuchowicz Spacetime. Universe 2020, 6, 124. [CrossRef]

28. Paston, S. Non-Relativistic Limit of Embedding Gravity as General Relativity with Dark Matter. Universe 2020, 6, 163. [CrossRef]

29. Berezin, V.; Dokuchaev, V.; Eroshenko, Y.; Smirnov, A. Formation and Clustering of Primordial Black Holes in Brans-Dicke Theory. Universe 2020, 6, 158. [CrossRef]

30. Bronnikov, K.; Bolokhov, S.; Skvortsova, M. Hybrid Metric-Palatini Gravity: Regular Stringlike Configurations. Universe 2020, 6, 172. [CrossRef]

31. Rubin, S.G.; Popov, A.; Petriakova, P.M. Gravity with Higher Derivatives in D-Dimensions. Universe 2020, 6, 187. [CrossRef]

32. Chernitskii, A.A. Gravitation in Unified Scalar Field Theory. Universe 2021, 7, 11. [CrossRef]

33. Saharian, A.A. Quantum Vacuum Effects in Braneworlds on AdS Bulk. Universe 2020, 6, 181. [CrossRef]

34. Dymnikova, I. The Higgs Mechanism and Spacetime Symmetry. Universe 2020, 6, 179. [CrossRef]

35. Mostepanenko, V.M.; Klimchitskaya, G.L. The State of the Art in Constraining Axion-to-Nucleon Coupling and Non-Newtonian Gravity from Laboratory Experiments. Universe 2020, 6, 147. [CrossRef]

36. Good, M.; Abdikamalov, E. Radiation from an Inertial Mirror Horizon. Universe 2020, 6, 131. [CrossRef]

37. Spirin, P. Gravitational Interaction of Cosmic String with Spinless Particle. Universe 2020, 6, 184. [CrossRef]

38. Fil'chenkov, M.; Laptev, Y. Quantization of Gravitationally Bound Systems. Universe 2021, 7, 30. [CrossRef]

39. Baryshev, Yu. Einstein's Geometrical versus Feynman's Quantum-Field Approaches to Gravity Physics: Testing by Modern Multimessenger Astronomy. Universe 2020, 6, 212. [CrossRef]

40. Alharthy, A.; Kassandrov, V.V. On a Crucial Role of Gravity in the Formation of Elementary Particles. Universe 2020, 6, 193. [CrossRef]

41. Zloshchastiev, K.G. Density Operator Approach to Turbulent Flows in Plasma and Atmospheric Fluids. Universe 2020, 6, 216. [CrossRef]

42. Nikonov, M.; Chekal, M.; Shirokov, S.; Baryshev, A.; Gorokhov, V. The Line-of-Sight Analysis of Spatial Distribution of Galaxies in the COSMOS2015 Catalogue. Universe 2020, 6, 215. [CrossRef]

43. Butuzova, M.S.; Pushkarev, A.B. Is OJ 287 a Single Supermassive Black Hole? Universe 2020, 6, 191. [CrossRef] 
44. Serov, V.D.; Roshchupkin, S.P.; Dubov, V.V. Resonant Effect for Breit-Wheeler Process in the Field of an X-ray Pulsar. Universe 2020, 6, 190. [CrossRef]

45. Gainutdinov, R.; Baryshev, Y. Relativistic Effects in Orbital Motion of the S-Stars at the Galactic Center. Universe 2020, 6, 177. [CrossRef]

46. Yelatontsev, V.A.; Roshchupkin, S.P; Dubov, V.V. Resonant Production of an Ultrarelativistic Electron-Positron Pair at the Gamma Quantum Scattering by a Field of the X-ray Pulsar. Universe 2020, 6, 164. [CrossRef]

47. Dokuchaev, V.I.; Nazarova, N.O. Visible Shapes of Black Holes M87* and SgrA*. Universe 2020, 6, 154. [CrossRef]

48. Larin, N.R.; Roshchupkin, S.P.; Dubov, V.V. Resonant Effects in a Photoproduction of Ultrarelativistic Electron-Positron Pairs on a Nucleus in the Field of the X-ray Pulsar. Universe 2020, 6, 141. [CrossRef]

49. Piotrovich, M.; Krasnikov, S.; Buliga, S.; Natsvlishvili, T. Possible Wormhole Candidates in Active Galactic Nuclei. Universe 2020, 6, 120. [CrossRef]

50. Doroshenko, D.V.; Roshchupkin, S.P.; Dubov, V.V. The Resonant Effect of an Annihilation Channel in the Interaction of the Ultrarelativistic Electron and Positron in the Field of an X-ray Pulsar. Universe 2020, 6, 137. [CrossRef]

51. Silant'ev, N.; Alekseeva, G.; Ananjevskaja, Y.; Novikov, V. Intensity and Polarization of Radiation Emerging from Rotating Accretion Disc with Increasing Height. Universe 2020, 6, 117 . [CrossRef]

52. Sizykh, G.K.; Roshchupkin, S.P.; Dubov, V.V. Resonant Ultrarelativistic Electron-Positron Pair Production by High-Energy Electrons in the Field of an X-ray Pulsar. Universe 2020, 6, 132. [CrossRef] 